\title{
Uniqueness of the Positive Energy Parafermion Field
}

\author{
Steven Robbins \\ Department of Mathematics, Massachusetts Institute of Technology, \\ Cambridge, Massachusetts, USA
}

Received April 5, 1974; in revised form May 23, 1974

\section{Introduction}

In 1953, Green [1] proposed that the usual anticommutation relations of Fermi-Dirac statistics be generalized. He proposed new relations which are satisfied by the Fermi-Dirac creation and annihilation operators but admit additional representations, each of which can also be given a particle interpretation. These particles are called parafermions.

For $n$ degrees of freedom it has been shown [4] that the representations of Green's relations are just the representations of $S O(2 n+1, R)$, which are well known. For infinitely many degrees of freedom the situation is more complicated. Reducible representations may be constructed by the use of Green's ansatz $[1 ; 2]$ from which certain standard irreducible representations are chosen. The uniqueness of these standard representations is usually discussed in terms of the existence of a unique vacuum vector which is annihilated by all annihilation operators. The purpose of this paper is to obtain uniqueness results relating to unitary invariance as was done for ordinary particles by Segal $[5$, Theorems 1,2,3] and Weinless [6, Theorem 2.1].

\section{Preliminaries}

A bounded representation of Green's parafermion relations is a triple $\{H, C, K\}$ where $H$ and $K$ are complex Hilbert spaces and $C$ is a complex linear map from $H$ into the bounded operators on $K$ satisfying

$$
\begin{gathered}
{\left[\left[C^{*}(z), C(y)\right], C(x)\right]=-2\langle x, z\rangle C(y),} \\
{[[C(z), C(y)], C(x)]=0 \quad x, y, z \in H .}
\end{gathered}
$$

$C^{*}(z)$ represents the adjoint of $C(z)$. 
The representation is said to be over the single particle space $H$ and have representation space $K$. Two representations over $H,\{H, C, K\}$ and $\left\{H, C^{\prime}, K^{\prime}\right\}$ are unitarily equivalent if there exists a unitary operator $U$ from $K$ onto $K^{\prime}$ such that $U C(z) U^{-1}=C^{\prime}(z)$, for $z \in H$.

A vector $v \in K$ is called cyclic for the representation if it is cyclic for the algebra $\boldsymbol{A}$ of finite linear combinations of products of operators in the form $C(z)$ or $C^{*}(z)$, i.e. if $\boldsymbol{A} v$ is dense in $K$. A representation is called the standard representation of order $p$ if there is a cyclic vector $v \in K$ such that

$$
\begin{aligned}
C^{*}(z) v & =0, \\
C^{*}(z) C(y) v & =\langle y, z\rangle p v \quad(y, z \in H) .
\end{aligned}
$$

In this case $p$ must be a positive integer and the standard representation of order $p$ is irreducible and unique up to unitary equivalence [2, p. B1158].

We will be considering continuous representations $\Gamma$ of the unitary group of $H$ on $K$. For these there exists a unique map $d \Gamma$ which takes a self-adjoint operator $A$ on $H$ into a self-adjoint operator $d \Gamma(A)$ on $K$ such that

$$
\Gamma\left(e^{i t A}\right)=e^{i t d \Gamma(A)} .
$$

If $d \Gamma(A) \geqq 0$ whenever $A \geqq 0$ we will write $d \Gamma \geqq 0$.

$P_{z}$ will denote the projection on the one dimensional space spanned by $z$, and $n(z)$ is defined by

$$
n(z)=\frac{1}{2}\left[C(z), C^{*}(z)\right] .
$$

$C(z)$ will be interpreted as creating a particle with wave function $z$ and $C^{*}(z)$ will be the corresponding annihilator. If $A$ is an observable on the single particle space $H$, i.e. a self-adjoint operator on $H$, then $d \Gamma(A)$ will be the corresponding observable on $K . n(z)+$ constant can be thought of as the number of particles with wave function $z$.

We will need the following lemma whose proof does not depend on the relations (1) and (2).

Lemma. Suppose $\{H, C, K\}$ is a bounded representation of Green's parafermion relations and $\Gamma$ is a representation of the unitary group of $H$ on $K$ satisfying

$$
\Gamma(U) C(z) \Gamma(U)^{-1}=C(U z)
$$

for all $z \in H$ and all unitaries $U$ on $H$. If $A$ is self-adjoint on $H$ and $z \in \operatorname{Dom}(A)$ then $\operatorname{Dom}(d \Gamma(A))$ is invariant under $C(z)$ and $C^{*}(z)$ and

$$
\begin{gathered}
{[d \Gamma(A), C(z)] \cong C(A z),} \\
{\left[d \Gamma(A), C^{*}(z)\right] \cong-C^{*}(A z) .}
\end{gathered}
$$


As a special case of this, when $\|z\|=1$,

$$
\begin{gathered}
{\left[d \Gamma\left(P_{z}\right), C(y)\right] \cong\langle y, z\rangle C(z),} \\
{\left[d \Gamma\left(P_{z}\right), C^{*}(y)\right] \cong-\langle z, y\rangle C^{*}(z),}
\end{gathered}
$$

which is equivalent to

$$
\begin{aligned}
e^{i t d \Gamma\left(P_{z}\right)} C(y) e^{-i t d \Gamma\left(P_{z}\right)} & =C\left(e^{i t P_{z}} y\right), \\
e^{i t d \Gamma\left(P_{z}\right)} C^{*}(y) e^{-i t d \Gamma\left(P_{z}\right)} & =C\left(e^{-i t P_{z}} y\right) .
\end{aligned}
$$

As a direct consequence of the relation (1),

$$
\begin{gathered}
{[n(z), C(y)]=\langle y, z\rangle C(z),} \\
{\left[n(z), C^{*}(y)\right]=-\langle z, y\rangle C^{*}(z),}
\end{gathered}
$$

and so when $\|z\|=1$,

$$
\begin{aligned}
e^{i t n(z)} C(y) e^{-i t n(z)} & =C\left(e^{i t P_{z}} y\right), \\
e^{i t n(z)} C^{*}(y) e^{-i t n(z)} & =C\left(e^{-i t P_{z}} y\right) .
\end{aligned}
$$

Thus for $\|z\|=1, e^{-i t d \Gamma\left(P_{z}\right)} e^{i t n(z)}$ commutes with all $C(y)$ and $C^{*}(y)$ and since (8) and (9) imply that $n(z)$ and $d \Gamma\left(P_{z}\right)$ commute, $n(z)-d \Gamma\left(P_{z}\right)$ commutes with all $C(y)$ and $C^{*}(y)$. For arbitrary $z \in H, z \neq 0$, we define $B(z)$ by

$$
B(z)=-2\left(\frac{n(z)}{\langle z, z\rangle}-d \Gamma\left(P_{z}\right)\right) .
$$

$B(z)$ is then a self-adjoint operator whose domain is invariant under and commutes with all elements of $A$. In the interesting cases $B(z)$ will in fact be independent of $z$. When the representation is irreducible it will be a scalar.

\section{Uniqueness Results}

Theorem 1 gives a general uniqueness result for parafermion fields. Theorems 2 and 3 extend this result along different lines. In the last section the possibility of obtaining a stronger theorem is discussed.

Theorem 1. Let $\{H, C, K\}$ be a bounded representation of Green's parafermion relations for which there exists a cyclic unit vector $v \in K$ and a representation $\Gamma$ of the unitary group of $H$ on $K$ such that

$$
\begin{aligned}
\Gamma(U) C(z) \Gamma(U)^{-1} & =C(U z), \\
\Gamma(U) v & =v, \\
d \Gamma & \geqq 0,
\end{aligned}
$$


for all $z \in H$ and all unitaries $U$ on $H$. Then $\{H, C, K\}$ is a finite direct sum of standard representations of distinct orders.

Proof of Theorem 1. $d \Gamma(A) v=0$ for all self-adjoint $A$ on $H$ since $e^{i t d \Gamma(A)} v=v$. By $(5)$,

$$
d \Gamma(I) C^{*}(z) v=C^{*}(z) d \Gamma(I) v-C^{*}(z) v=-C^{*}(z) v .
$$

Since $d \Gamma(I) \geqq 0, C^{*}(z) v=0$ for all $z \in H$. Now suppose $\langle y, z\rangle=0$.

Since $d \Gamma\left(P_{y}\right) \geqq 0$,

$$
\begin{aligned}
d \Gamma\left(P_{y}\right) C^{*}(y) C(z) v & =-C^{*}(y) C(z) v+C^{*}(y) C(z) d \Gamma\left(P_{y}\right) v \\
& =-C^{*}(y) C(z) v .
\end{aligned}
$$

$$
C^{*}(y) C(z) v=0 \quad \text { when }\langle y, z\rangle=0 .
$$

We will show that the operator $B(z)$ defined by $(10)$ is independent of $z$. It is sufficient to consider the case in which $z$ is a unit vector. Since $d \Gamma\left(P_{z}\right) v=0$, and $C^{*}(z) v=0$,

$$
B(z) v=C^{*}(z) C(z) v .
$$

Since $B(z)$ commutes with $C(z)$ and $C^{*}(z)$,

If $D \in A$, then

$$
B(z)^{k} v=\left(C^{*}(z) C(z)\right)^{k} v .
$$

Therefore,

$$
\begin{aligned}
& B(z) D v=D B(z) v=D C^{*}(z) C(z) v \in A v \\
&\left\|B(z)^{k} D v\right\|=\left\|D B(z)^{k} v\right\| \leqq\|D\|\left\|C^{*}(z) C(z)\right\|^{k}\|v\| . \\
& \sum_{k}\left\|\frac{B(z)^{k} t^{k} D v}{k !}\right\|
\end{aligned}
$$

converges and so each element of $\boldsymbol{A} v$ is an analytic vector for $B(z)$. We will be done if we show that $B(z) v$ is independent of $z$, for from this it follows that if $D \in A, B(z) D v=D B(z) v$ is independent of $z$ and thus $B(z)$ is independent of $z$ on a dense invariant set of analytic vectors.

We show that $B(z) v$ is independent of $z$ by using (14). If $y$ and $z$ are unit vectors then

$$
\begin{aligned}
C^{*}(z) C(y) v & =C^{*}\left(P_{y} z\right) C(y) v \\
& =\langle y, z\rangle C^{*}(y) C(y) v \\
& =\langle y, z\rangle B(y) v \\
C^{*}(z) C(y) v & =C^{*}(z) C\left(P_{z} y\right) v \\
& =\langle y, z\rangle C^{*}(z) C(z) v \\
& =\langle y, z\rangle B(z) v .
\end{aligned}
$$

Thus $B(y) v=B(z) v$ when $\langle y, z\rangle \neq 0$. For $\langle y, z\rangle=0$ we obtain the result by observing that if $w=y+z, B(y) v=B(w) v=B(z) v$. 
Since $\Gamma(U) d \Gamma\left(P_{z}\right) \Gamma(U)^{-1}=d \Gamma\left(P_{U z}\right)$ and

$$
\Gamma(U) n(z) \Gamma(U)^{-1}=n(U z), \quad \Gamma(U) B(z) \Gamma(U)^{-1}=B(U z)=B(z) .
$$

Thus, $\Gamma(U)$ commutes with $B(z)$.

Let $B=B(z)$. Assume $\|z\|=1$ and $d \Gamma\left(P_{z}\right) w=0$. Then $C^{*}(z) w=0$ and $B w=C^{*}(z) C(z) w$. Let $w_{m}=C(z)^{m} w$. From (1) it follows that

Therefore

$$
\begin{aligned}
C^{*}(z) w_{m} & =m(B-m+1) w_{m-1}, \\
\left\|w_{m}\right\|^{2} & =\left\langle w_{m-1}, C^{*}(z) w_{m}\right\rangle \\
& =m\left\langle w_{m-1},(B-m+1) w_{m-1}\right\rangle \\
& =m !\langle w,(B-m+1)(B-m+2) \ldots(B-1) B w\rangle .
\end{aligned}
$$

$$
\langle B(B-1)(B-2) \ldots(B-m) w, w\rangle \geqq 0, \quad \text { for } \quad m \geqq 0 .
$$

Let $K_{0}$ be the Hilbert space spanned by $B^{k} v, k=0,1,2, \ldots B$ is bounded on $K_{0}$ since if $Q$ is any polynomial in $B$,

$$
\begin{aligned}
B Q v & =Q B v=Q C^{*}(z) C(z) v=C^{*}(z) C(z) Q v, \\
\|B Q v\| & \leqq\left\|C^{*}(z) C(z)\right\|\|Q v\| .
\end{aligned}
$$

Now consider $B$ as a bounded operator from $K_{0}$ into itself. If $w \in K_{0}$, $d \Gamma\left(P_{z}\right) w=0$ since $B$ commutes with all $\Gamma(U)$. Thus (15) holds for all $w \in K_{0}$ and so on $K_{0}, B(B-1)(B-2) \ldots(B-m) \geqq 0$ for every integer $m$. Thus the spectrum of $B$ just consists of integral eigenvalues. Let $K_{0}(m)=\left\{w \in K_{0}: B w=m w\right\}$. Let $P_{m}$ be the projection on the closure of $\boldsymbol{A} K_{0}(m)$. If $w \in K_{0}(m), w=\Sigma a_{k} B^{k} v$ and

$$
w=P_{m} w=P_{m} \Sigma a_{k} B^{k} v=\Sigma a_{k} B^{k} P_{m} v=\left(\Sigma a_{k} m^{k}\right) P_{m} v .
$$

So $K_{0}(m)$ either has dimension 0 or 1 . Let $S=\left\{m: K_{0}(m)\right.$ has dimension 1$\}$. Let $v_{m}=\frac{P_{m} v}{\left\|P_{m} v\right\|}, m \in S$. Define $K^{m}$ to be the closure of $\boldsymbol{A} K_{0}(m)$. If $m \neq s$ then $K^{m}$ and $K^{s}$ are orthogonal since if $w \in A K_{0}(m)$ then $B w=m w$. $K=\sum_{m \in S} \oplus K^{m}$ and $C^{*}(z) v_{m}=0$ since $\left[C^{*}(z), P_{m}\right]=0$.

$$
\begin{aligned}
C^{*}(y) C(z) v_{m} & =P_{m} C^{*}(y) C(z) v \\
& =P_{m}\langle z, y\rangle B(z) v=\langle z, y\rangle B P_{m} v \\
& =m\langle z, y\rangle v_{m}, \\
\Gamma(U) B^{k} v & =B^{k} \Gamma(U) v=B^{k} v .
\end{aligned}
$$

So $\Gamma(U) v_{m}=v_{m}$. Since each $P_{m}$ commutes with $C(z)$ and $C^{*}(z)$ the representation is the desired direct sum. This completes the proof of Theorem 1. 
Theorem 2. In Theorem 1, Eq.(12) can be replaced by the weaker condition: There exists a non-negative self-adjoint operator $A$ on $H$ whose discrete spectrum does not contain 0 such that $\Gamma\left(e^{i t A}\right) v=v$.

To prove Theorem 2 we will use the following properties of $d \Gamma$. Suppose $A$ and $B$ are commuting self-adjoint operators on $H$. Then

a) $d \Gamma(A)$ and $d \Gamma(B)$ commute.

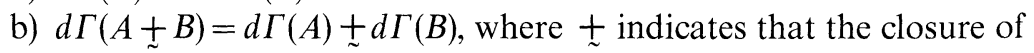
the sum should be taken.

c) $d \Gamma(\alpha A)=\alpha d \Gamma(A)$ if $\alpha \neq 0$ and is real.

d) If $d \Gamma \geqq 0$ and $A \geqq B$ then $d \Gamma(A) \geqq d \Gamma(B)$.

Let $A=\int \lambda E(d \lambda)$ be the spectral resolution of $A$. Define $P_{k}=E\left(I_{k}\right)$ where $I_{k}$ is the interval $\left(\frac{1}{k}, \infty\right)$. Then $A \geqq \frac{1}{k} P_{k}$ so by $\left.d\right)$ above, $d \Gamma(A)$ $\geqq \frac{1}{k} d \Gamma\left(P_{k}\right) \geqq 0$ and $d \Gamma(A)$ and $\frac{1}{k} d \Gamma\left(P_{k}\right)$ commute. Since $d \Gamma(A) v=0$ we have also $\frac{1}{k} d \Gamma\left(P_{k}\right) v=0$ so $\Gamma\left(e^{i t P_{k}}\right) v=v$. Since 0 is not in the discrete spectrum of $A, P_{k} \rightarrow I$ strongly as $k \rightarrow \infty$.

$$
e^{i t P_{k}}=1+\left(e^{i t}-1\right) P_{k}
$$

so $e^{i t P_{k}} \rightarrow e^{i t}$ strongly for each $t$. Therefore $\Gamma\left(e^{i t P_{k}}\right) \rightarrow \Gamma\left(e^{i t}\right)$ strongly so $\Gamma\left(e^{i t}\right) v=v$. Thus, $d \Gamma(I) v=0$.

If $E$ is any bounded, non-negative self-adjoint operator on $H$ then $\|E\| I \geqq E \geqq 0$ and so $d \Gamma(\|E\| I) \geqq d \Gamma(E) \geqq 0$. Thus $d \Gamma(E) v=0$. By separating a bounded self-adjoint operator into positive and negative parts we obtain $d \Gamma(E) v=0$ for all bounded self-adjoint operators on $H$. From this it follows that $\Gamma(U) v=v$ for all unitaries $U$ on $H$.

Theorem 3. Let $\{H, C, K\}$ be a bounded representation of Green's parafermion relations for which there exists a cyclic vector $v \in K$ and $a$ representation of the unitary group of $H$ on $K$ such that for all $z \in H$ and all unitaries $U$ on $H$,

$$
\begin{aligned}
\Gamma(U) C(z) \Gamma(U)^{-1} & =C(U z), \\
\Gamma(U) v & =v,
\end{aligned}
$$

there exists a non-negative self-adjoint operator $A$ on $H$ other than 0 such that $d \Gamma(A) \geqq 0$, and either the representation is irreducible or the operator $A$ above is not a multiple of the identity. Then $\{H, C, K\}$ is a direct sum of standard representations of distinct orders.

The only use of the positivity condition (13) in Theorem 1 was to prove that $C^{*}(y) v=0$ and $C^{*}(y) C(z) v=0$ for orthogonal $y$ and $z$. These will now be proved under the weaker hypotheses.

By (16) and (17), $\left\|C^{*}(z) v\right\|$ and $\left\|C^{*}(y) C(z) v\right\|$ are independent of $y$ and $z$ when $y$ and $z$ are orthogonal unit vectors. We can show that these are zero by finding values of $y$ and $z$ for which they are arbitrarily small. 
Since $\|C(y)\|=\left\|\Gamma(U) C(y) \Gamma(U)^{-1}\right\|=\|C(U y)\|,\|C(y)\|$ depends only on $\|y\|$ and since $C(y)$ is a linear function of $y$,

$$
\|C(y)\|=\|C\|\|y\| \text {. }
$$

In a similar manner,

$$
\left\|C^{*}(y)\right\|=\left\|C^{*}\right\|\|y\| .
$$

Choose $r \neq 0$ in the spectrum of $A$. For each $\varepsilon>0$ there is a unit vector $z \in K$ such that $\|(A-r) z\|<\varepsilon$. By $(7), d \Gamma(A) C^{*}(z) v=-C^{*}(A z) v$ so

$$
\begin{aligned}
0 & \leqq\left\langle d \Gamma(A) C^{*}(z) v, C^{*}(z) v\right\rangle \\
& \leqq-\left\langle C^{*}(A z) v, C^{*}(z) v\right\rangle \\
& \leqq-\left\langle C^{*}((A-r) z) v, C^{*}(z) v\right\rangle-r\left\langle C^{*}(z) v, C^{*}(z) v\right\rangle \\
& \leqq\left\|C^{*}(z) v\right\|\left\|C^{*}((A-r) z) v\right\|-r\left\|C^{*}(z) v\right\|^{2} \\
\left\|C^{*}(z) v\right\| & \leqq\left\|C^{*}((A-r) z) v\right\| / r \\
\left\|C^{*}(z) v\right\| & \leqq \varepsilon\left\|C^{*}\right\|\|v\| / r .
\end{aligned}
$$

Thus, $C^{*}(z) v=0$ for all $z$.

We will now show that $C^{*}(y) C(z) v=0$ for orthogonal $y$ and $z$. We first treat the case in which $A$ is not a multiple of the identity. Choose two distinct numbers $r$ and $s$ in the spectrum of $A$ and assume $r<s$. For each $\varepsilon>0$ there exist orthogonal unit vectors $y$ and $z$ such that both $\|(A-r) z\|$ and $\|(A-s) y\|$ are less than $\varepsilon$. Then if $w=C^{*}(y) C(z) v$, (6) and (7) imply

$$
\begin{aligned}
& d \Gamma(A) w=C^{*}(y) C(A z) v-C^{*}(A y) C(z) v . \\
& 0 \leqq\langle d \Gamma(A) w, w\rangle \\
& \leqq\left\langle C^{*}(y) C(A z) v, w\right\rangle-\left\langle C^{*}(A y) C(z) v, w\right\rangle \\
& \leqq r\|w\|^{2}+\left\langle C^{*}(y) C((A-r) z) v, w\right\rangle \\
&-s\|w\|^{2}-\left\langle C^{*}((A-s) y) C(z) v, w\right\rangle \\
& \leqq(r-s)\|w\|^{2}+2\|w\|\left\|C^{*}\right\|\|C\|\|v\| \varepsilon .
\end{aligned}
$$

Thus, $\|w\| \leqq 2 \varepsilon\|C\|\left\|C^{*}\right\|\|v\| /(s-r)$ and so $C^{*}(y) C(z) v=0$ for all orthogonal pairs of unit vectors $y$ and $z$ in $H$.

This leaves the case in which the representation is irreducible. In this case $d \Gamma\left(P_{z}\right)-n(z)$ is a scalar. Since $d \Gamma\left(P_{z}\right) v=0$ and $C^{*}(z) v=0$ for all $z \in H, C^{*}(z) C(z) v=\|z\|^{2} B(z) v$ where $B(z)$ is now a scalar depending only on $z$. As in the proof of Theorem 1,(15) with $v$ replacing $w$ shows that $B(z)$ must be an integer when $z$ is a unit vector. Since $B(z)$ is continuous on the unit ball of $H, B(z)$ must be a constant $p$ on this 
ball, and thus,

$$
C^{*}(z) C(z) v=p\|z\|^{2} v,
$$

for all $z \in H$. Applying (18) for $z=x+y$ and $z=x+i y$ and subtracting gives $C^{*}(x) C(y) v=p\langle y, x\rangle v$. This completes the proof of Theorem 3 .

\section{Concluding Remarks}

In Theorems 2 and 3 we showed that each of (12) and (13) could separately be replaced by weaker conditions. In [3, pp. 70-75] examples were given that show that these conditions could not be eliminated and that a simultaneous weakening of both conditions as in [6, Theorem $2.1]$ is impossible. Theorem 2 gives a maximal weakening of ${ }^{-}(12)$. In Theorem 3 the weakened condition is unaesthetic mathematically and inconvenient physically since for reducible representations it requires that the operator $A$ is not a multiple of the identity. This case is physically interesting because $d \Gamma(I)$ represents the number operator on $K$. We would like to have a theorem which states that subject to (11) and (12) it is sufficient to have a positive number operator. It can be easily shown that in this case the condition $d \Gamma(I) \geqq 0$ is equivalent to $C^{*}(z) v=0$ for all $z \in H$, a condition much easier to establish. We have found an example of a direct sum of two irreducible non-standard parafermion fields for which (11), (12) and this condition are satisfied. This shows that Theorem 3 also gives a maximal weakening of (13). We would like to thank Project MAC of the Massachusetts Institute of Technology for making available MACSYMA, their symbolic manipulation system. It was with the use of this system that the latter example was found.

\section{References}

1. Green,H.S.: Phys. Rev. 90, 270-273 (1953)

2. Greenberg, O.W., Messiah,A. M.L.: Phys. Rev. 138, B1155-1167 (1965)

3. Robbins, S.: Parastatistics in Quantum Field Theory, Ph. D. Dissertation, M. I. T., June, 1973

4. Ryan,C., Sudarshan,E.C.D.: Nucl. Phys. 47, 207-211 (1963)

5. Segal, I. E.: Illinois J. Math. 6, 500-523 (1962)

6. Weinless, M.: J. Funct. Anal. 4, 350-379 (1969)

Communicated by A. S. Wightman

Steven Robbins

2-114

Department of Mathematics

Massachusetts Institute of Technology

Cambridge, Massachusetts 02139, USA 